\title{
Effects of Postpartum Family Planning Counseling on Contraceptives Among Women Attending a General Hospital in the Gambia: A Randomized Trial
}

Jainaba Sey-Sawo ( $\sim$ jsey@utg.edu.gm )

University of the Gambia https://orcid.org/0000-0003-1532-2865

Florence 0. Adeyemo

University of Benin School of Basic Medical Science

Obehi H. Okojie

University of Benin

\section{Research}

Keywords: Postpartum Family Planning, Counseling, Contraceptives, Women, The Gambia

Posted Date: June 2nd, 2021

DOI: https://doi.org/10.21203/rs.3.rs-535193/v1

License: (c) (i) This work is licensed under a Creative Commons Attribution 4.0 International License.

Read Full License 


\section{Effects of Postpartum Family Planning Counseling on Contraceptives among Women attending a General Hospital in the Gambia: A Randomized trial}

Jainaba Sey-Sawo ${ }^{1}$, Florence O. Adeyemo ${ }^{2} \&$ Obehi H. Okojie ${ }^{3}$

1. Senior Lecturer, Department of Nursing and Reproductive Health, University of the Gambia, The Gambia

2. Professor, Department of Nursing, University of Benin, Edo State, Nigeria

3. Professor, Department of Community Health, University of Benin, Edo State, Nigeria

* Corresponding - email- jsey@ utg.edu.gm; phone (+220) 3650105

\section{Plain English Summary}

Family planning counselling helps clients choose and use family planning methods that suit clients' needs. The contraceptive uptake rate is very low in the Gambia. Therefore, the study aimed to evaluate the effectiveness of postpartum family planning counseling among women attending a general hospital in the Gambia. The intervention site was Bansang General Hospital (BGH) while Soma Health Center (SHC) was the comparison group A total 674 women were recruited and contraceptives counseling sessions were conducted three times during a six week period using contraceptive methods and posters at the intervention site. Women that delivered at SHC had no family planning counseling but had a health talk on umbilical care. The questionnaire assessed socio-demographic, knowledge, attitude and intention to utilize a contraceptive method and data were collected on face-to-face interviews in the three most spoken local languages; Wollof, Mandinka and Fulla.

Results indicated there was no statistically significant difference in participants' sociodemographic variables between the two study groups except for ethnicity, marital status and employment. Similarly, no significant difference was observed at baseline. However at post 
intervention, a statistically significant difference was noticed on knowledge, attitude, and intention between the intervention and comparison groups $(\mathrm{p}<0.05)$.

In conclusion, the study has highlighted the need to conduct a structured and regular family planning counseling in order to increase women's knowledge, attitude, and intention and subsequently contraceptive uptake. This will help to address the already low contraceptive uptake and thus reduce maternal mortality and morbidity in the country.

\section{Abstract}

\section{Background}

Postpartum Family Planning (PPFP) counseling is a critical component of maternal health care that has the potential of meeting women's desire for contraception. The study aimed to evaluate the effectiveness of postpartum family planning counseling among women attending a general hospital in the Gambia.

\section{Methods}

The study used a quasi-experimental design with two groups; the intervention and the comparison group. A sample size of 674 participants was determined by a formula for comparison between two groups. The questionnaire was developed based on a literature review and was pre-tested on $10 \%$ of the total study sample size (68). A reliability of 0.731 was obtained. Systematic random sampling method was employed to select those who met the inclusion criteria. Data were collected on face-to-face interviews in the Wollof, Mandinka and Fulla local languages with a $100 \%$ response rate at baseline and $96 \%$ at posttest. The intervention strategy was family planning counseling using the GATHER approach. Data were analyzed using SPSS version 21.00 and statistical analysis included both descriptive and inferential methods. Ethical approval was obtained from the Research and Ethic Committee, School of Basic Medical Sciences, College of Medicine, University of Benin (CMS/REC/2017/017) and the Gambia Government/Medical Research Council Laboratories Joint Ethics Committee (R017016Av1.1). 


\section{Results}

Baseline respondents' socio-demographic characteristics revealed that the two groups had similar characteristics and were therefore comparable $(\mathrm{P}>0.05)$. A statistically significant difference existed on knowledge, attitude, and intention between the intervention and comparison groups at post intervention $(\mathrm{p}<0.05)$ while no significant difference was observed at baseline. Significant gains were achieved in the intervention group in terms of knowledge, attitude, and intention post intervention.

\section{Conclusion}

The present study therefore highlights the need to conduct family planning counseling during the immediate postpartum period, particularly before discharge of women from the hospital so as to ensure improved contraceptive uptake, prevent unwanted pregnancy and closely spaced pregnancy and thus improve maternal health outcomes.

Keywords: Postpartum Family Planning, Counseling, Contraceptives, Women, The

\section{Gambia}




\section{Introduction}

Postpartum Family Planning (PPFP) counseling is a critical component of maternal health care that has the potential to meet women's desire for contraception, and hence improve contraceptive uptake. PPFP is defined as the prevention of unintended pregnancy and closely spaced pregnancies through the first 12 months following childbirth ${ }^{1}$. Globally, nearly $65 \%$ of women in their first postpartum year have an unmet need for family planning services ${ }^{2}$, with more than 220 million women in developing countries, mainly in South Asia and sub-Saharan Africa ${ }^{3}$. In India, $65 \%$ of women in the first year postpartum have an unmet need for family planning ${ }^{4}$. High unmet need for family planning (FP) contributes to low contraceptive use. In addition, PPFP usage varies in sub-Saharan countries, and is $40 \%$ in Zambia, $25 \%$ in Kenya, $20 \%$ in Tanzania, $15 \%$ in Nigeria and less than $10 \%$ in Ethiopia ${ }^{5}$. In The Gambia, the unmet needs stand at $25.8 \%$ 6 , and prevalence of contraceptives use among women is $9 \%$. However, PPFP usage is unknown and is believed to be low as well.

The goals of contraceptive counseling are to educate women about contraception, discuss current and future contraceptive needs, and select a contraceptive modality, if needed, thereby avoiding the risks of unintended pregnancy ${ }^{7}$. Evidence indicates that structured counseling both protects women's rights to an informed and voluntary decision regarding their reproductive choices and improves the use of modern contraception methods ${ }^{8}{ }^{9}$ ). The largest population of women with an unmet need for contraception is found among those in their first year after childbirth ${ }^{10}$. Studies reported that the rate of pregnancy within the postpartum period is $6-40 \%{ }^{11}$. 
The level of women's knowledge has a significant effect on future use and non-use of postpartum contraception. Researchers found that ${ }^{12}$, knowledge, and awareness on contraceptive is high among the Nigerian population. However, this awareness has not been translated into increased contraceptive use, and so contraceptive prevalence has remained low. Furthermore, a cross-sectional observational study conducted among postpartum women attending Kathmandu Medical College Teaching Hospital, revealed that contraceptive awareness and knowledge among the postpartum women was high but their usage was low ${ }^{13}$.

Attitude is the most difficult part to measure as it is characterized in a very abstract way. In Ebonyi State, Nigeria, researchers found postpartum women to have favorable attitude towards contraception $^{14}$. A study in Iran revealed that among postpartum women the intervention group had higher positive attitude towards contraceptives than their counter parts in the control group $(\mathrm{p}<0.05)^{15}$.

A study conducted in Uganda revealed that $71.4 \%$ of women in the control and $87 \%$ in the intervention had intention to use a modern contraceptive method following counseling on Postpartum Family Planning ${ }^{16}$. Similarly studies from Nigeria showed that most women intended to use a method of postpartum contraception ${ }^{17}$ while in Ohio, most post-partum women (91\%) intended to use contraception before their discharge following delivery at a large University hospital $^{18}$.

In The Gambia, family planning services have been free of charge and available in all public health facilities since 1975, yet, the contraceptive prevalence rate (CPR) is showing a downward 
trend in married women aged 15-49 years for various reasons such as low educational level and

religious barrier ${ }^{6}$. Hence, contraceptive counseling during the postpartum period is of paramount importance to improve knowledge, attitude and intention and the use of modern contraception methods which help to prevent or delay a subsequent pregnancy after a live birth. A literature search revealed that no published study on PPFP counselling on the topic was found credited to the country. Therefore, the aim of this study was to evaluate the effects of postpartum family planning counseling on contraceptive knowledge, attitude and intention to use among women attending health facilities in The Gambia.

\section{Methodology}

A hospital based quasi experimental study design with a control group was used for this study.

\section{Study Setting}

The study was conducted in two health facilities; Bansang General Hospital (BGH) was the intervention facility located in Central River Region, rural Gambia, about $300 \mathrm{~km}$ from the capital city Banjul. Furthermore, in order to prevent contamination, the control group was observed at Soma Health Center (SHC) located in the Lower River Region, about 180km from the capital.

\section{Sample Size and Sampling Technique}

The sample size estimation of this study was determined by using a formula for sample size calculation for comparison between two groups when endpoint is quantitative data ${ }^{19}$. Using a prevalence of $57 \%$ and $49 \%$, women that accepted contraceptives following postpartum family 
planning counselling in Rwanda ${ }^{20}$, the sample size of this study was determined by the formula below:

$$
n=\frac{2 \times\left(z_{\alpha}+z_{\beta}\right)^{2} \times p \times(1-p)}{\left(p_{0}-p_{1}\right)^{2}} ; \text { Where, } p=\frac{p_{0}+p_{1}}{2}
$$

Thus;

for $\alpha=0.05 ; \beta=0.20 ; \mathrm{p}_{0}=57 \%$ and $\mathrm{p}_{1}=49 \%$;

$n=\frac{2 \times(1.96+0.84)^{2} \times 0.53 \times(0.47)}{(0.08)^{2}} ;$ where $p=\frac{0.57+0.49}{2}$

The calculated sample size $(n)=612$; provision for $10 \%$ non response rate was made and the final sample size was 674 .

\section{The Intervention Group}

Inclusion criteria for women in the intervention group was based on all those who delivered at Bansang General Hospital (BGH) with a live baby on day 1, aged between 13-49 years, and delivered in BGH during/between the months of November, 2017 and May, 2018 and were receiving postnatal care and attending infant welfare clinic at the $\mathrm{BGH}$ or within its catchment area. Exclusion was based on severe chronic illness of mother (mental, cardiac, cancer, liver, kidney), women with postpartum hemorrhage, infection and fever $>38^{\circ} \mathrm{c}$.

\subsubsection{The Control Group}

In order, to minimize contamination, the control group was at Soma Health Center (SHC) located in the Lower River Region, which is about 180 kilometers from the intervention site. Furthermore, women in this group included those who delivered at the health center with a live 
baby, on day 1, aged between 13-49 years, and delivered in SHC during/between the months of November, 2017 and May, 2018 and were receiving postnatal care and attending infant welfare clinic at the SHC or within its catchment area. Exclusion was based on severe chronic illness of mother (mental, cardiac, cancer, liver, kidney); women with postpartum hemorrhage, infection and fever $>38^{\circ} \mathrm{c}$.

\section{Study Tool}

A questionnaire was developed by the Principal Investigator based on literature review to assess women's knowledge, attitude, and intention towards postpartum family planning methods. The research objectives guided the development of the questionnaire. The questionnaire consisted of 37 closed ended questions. It was divided into five sections as follows: Section A; this elicited the socio-demographic characteristics of respondents. Section B obtained Knowledge of family planning. Two points were given to any correct answer, and zero point to any wrong or unknown answer. The total possible scores for this part of the questionnaire ranged from 0 to 54 . Scores from 0-17 was considered poor knowledge, and 18-54 good knowledge. Section C addressed postpartum women's contraceptive attitudes related factors were measured by 9 items on a five point Likert scale ranging from strongly agree $=5$, agree $=4$, don't know $=3$, disagree $=2$, strongly disagree $=1$. The total possible score for this part of the questionnaire ranged from 9 to 45 . Scores from 9-27 was considered negative, $28-45$ was positive. Finally Section D looked at intention to utilize contraceptives has 8 items on a five point Likert scale ranging from agree strongly $=5$, agree somewhat $=4$, don't know $=3$, disagree somewhat $=2$, and disagree strongly $=1$. This section determined if the woman intended to use modern contraceptive. The total possible score for this part of the questionnaire ranged from 8 to 40 . Scores from 8-25 was considered low, and 26-40 
was high. Each subscale was calculated separately, and therefore four different scores were obtained for each subject.

\section{Intervention Plan}

The primary goal of the intervention was to increase contraceptive use (this will be discussed in the follow up paper) among postpartum women, thus preventing unintended and closely spaced pregnancy. This goal was accomplished through contraceptive counseling designed to improve knowledge, attitude, and intention

Counseling was provided three times; day one before hospital discharge. This was conducted before or immediately after the medical wards rounds using the GATHER (Greet, Ask, Tell, Help, Explain and Return) approach ${ }^{21}$.

The second counseling was conducted on the $9^{\text {th }}$ day at the post-natal clinic, before or after the change of card (women came for a change of card, from antenatal to infant welfare card. This is because the child is given a name on the $8^{\text {th }}$ day in The Gambia). It is a cultural practice during the first week of postpartum for women stay indoors and only goes to the hospital if the child is sick. The second counseling session was conducted using the same GATHER approach, contraceptive poster and sample contraceptive methods as the first session.

The third counseling session was carried on the $40^{\text {th }}$ day at the Infant Welfare Clinic (e.g. while the woman was waiting for, or immediately after the baby received immunization). This session was conducted in the same way and manner as the first and second counseling sessions. 


\section{Post Intervention}

Regarding the post intervention, the same questionnaire was administered to women in both groups at the sixth week to assess knowledge, attitude, and intention to utilize modern contraception. Similarly, at the end of the first six weeks both groups were assessed to determine the level of knowledge, attitude, and intention to utilize modern contraception

\section{Reliability}

The study interview schedule was pre-tested tested using $10 \%$ of the total study participants (68), from two different health Facilities (34 for each Facility), on participants that had similar inclusion criteria as those that participated in the study. In this study, the reliability was 0.731. The questionnaire was modified based on the pre-test results.

\section{Data Collection}

Data was collected using interviewer administered questionnaires by trained 8 practicing nursemidwives who were trained as research assistants, they were fluent in at least two of the three languages (Wolof, Mandinka and Fula) spoken in the study sites. The baseline data were collected through face-to-face interviews and the questionnaire was retrieved immediately, thus a $100 \%$ return rate was obtained for both groups.

Data were collected from November, 2017 to May, 2018 in the following order: November December 2017 was used to collect baseline data, and December 2017-February-2018 post -test data. Analysis of data included both descriptive and inferential statistics. Data was presented using frequency tables, and summary statistics. Statistical tests were done using Chi-squared tests, t-tests and binary logistic regression. Statistical significance was set at $\mathrm{p}<0.05$. 
Results:

Table 1 shows the socio-demographic characteristics of the study participants. There was no statistically significant difference between the intervention and control groups in terms of their socio-demographic characteristics except for ethnicity, marital status and employment. More than a third of participants in both intervention and control groups had no formal education.

It was observed that at baseline there was no statistically significant difference between the two groups except for knowledge on types of contraceptives. Similarly, there was no significant difference in mean knowledge score between the intervention and the comparison group. $(\mathrm{p}=0.860)$. However, at post intervention there was a statistically significant difference in terms of knowledge on the entire domain measured and the mean knowledge score $(\mathrm{p}=0.000)$ as shown in Table 2.

Table 3 shows that the intervention group, a lesser proportion of respondents (18.4\%) had good knowledge at baseline and a higher proportion (61.3\%) had good knowledge post intervention, thus a difference of $42.9 \%$ was gained. Regarding the comparison group, a lesser proportion of respondents $(14.8 \%)$ had good knowledge at baseline and a greater proportion $(25.2 \%)$ had good knowledge post intervention, thus a difference of $10.4 \%$ was achieved. Therefore, the difference between the two study groups was $32.5 \%$.

Table 4 revealed that the respondents from the intervention group were 4.694 (95\% CI: 3.3566.566) times more likely to have good knowledge of contraceptives than their counterparts in the comparison group. This was statistically significant $(\mathrm{p}=0.000)$. 
Table 5 shows that at baseline there was no statistically significant difference between the two groups for attitudes towards contraceptives and the mean attitude score $(p>0.05)$.. However, at post intervention there was a statistically significant difference in terms of attitude on the entire domain measured and the mean attitude score $(\mathrm{p}=0.000)$.

Table 6 shows that in the intervention group, a lesser proportion of respondents $(57.1 \%)$ had positive attitude at baseline and a higher proportion (79.0\%) had positive attitude post intervention, thus a difference of $21.9 \%$ was gained. Regarding the comparison group, a lesser proportion of respondents $(53.7 \%)$ had positive attitude at baseline and a greater proportion (58.2\%) had positive attitude post intervention, thus a difference of $4.5 \%$ was registered. Therefore, the difference between the two study groups was $17.4 \%$.

Table 7 revealed that the respondents from the intervention group were 2.712 times (95\% CI: 1.913- 3.843) more likely to have positive attitude to contraceptive use than their counterpart in the comparison group. This was statistically significant $(\mathrm{p}=0.000)$. 
According to Table 8, there was no statistically significant difference between the two groups in terms of intention to utilize contraceptives between the two groups and the mean intention score at baseline $(\mathrm{p}>0.05)$. However, at post intervention there was a statistically significant difference on intention on the entire domain measured and the mean intention score $(\mathrm{p}=0.000)$.

Table 9 revealed that in the intervention group, a lesser proportion of respondents $(29.0 \%)$ had low intention at baseline and a higher proportion $(98.4 \%)$ had high intention post intervention, thus a difference of $69.4 \%$ was gained. Regarding the comparison group, a higher proportion of respondents $(28.8 \%)$ had low intention at baseline and a lesser proportion $(20.5 \%)$ had low intention post intervention, thus a difference of $-8.3 \%$ was registered. Therefore, the difference between the two study groups was $77.7 \%$.

Table 10 above shows that revealed that the respondents from the intervention group were 36.419 times (95\% CI: 22.305- 59.464) more likely to have high intention to contraceptive use than their counterpart in the comparison group. This was statistically significant $(\mathrm{p}=0.000)$.

\section{DISCUSSION}

In this study, the mean ages of participants were 25.4 and 25.8 years in the intervention and comparison groups respectively. These were not statistically significant $(\mathrm{p}>0.05)$. This shows that participants had a similar characteristic in terms of age, were young and within their reproductive ages. This age group was a very good target for postpartum contraceptive uptake for the increase of contraceptive prevalence rate and reduction of unwanted and of closely space pregnancy. This age characteristic is consistent with a study conducted in Kenya which revealed that the mean age of post-partum women was 26 years ${ }^{22}$. Similarly, an Indian study found that 
the mean age of its participants was 25.6 years $^{23}$. A greater proportion of women were multiparous in both groups. A possible explanation for the multiparity among women could be the desire for a male child and large family size for economic and security reasons. This means that the study participants may want to use contraceptives for spacing but not to limit family size. The health implication of high parity could be associated with increased adverse obstetric outcomes and socio-economic implications for the family. This is congruent with a study which reported similar findings in The Gambia ${ }^{24}$. In this study, a higher proportion of participants had elementary education in the intervention group while most had no formal education in the comparison group. The difference in educational levels was not statistically significant(p>0.05) and could be attributed to the fact that most rural Gambian families prefer to send the male child to formal school as opposed to the girl child and secondly, it could be due to early marriage of the girl child. This implies that respondents might be unable to make an informed decision with regards to their reproductive health matters such as marriages, family size and contraceptive use. To avert this state of affairs improving girls' education and empowering them to make informed decisions in this part of the country should be a government priority. In a similar study found that about $41.7 \%$ of postpartum women were uneducated in rural Tigray region, northern Ethiopia ${ }^{25}$.

\section{Participants' Knowledge on Contraceptives}

At baseline, majority of respondents had poor contraceptive knowledge level in both the intervention and comparison groups. There was no significant difference in knowledge between the two study groups. This is in line with a Zimbabwean study on contraceptive counselling 
among HIV positive mothers, which showed that there was no significant difference in knowledge between the intervention and comparison groups at baseline $(\mathrm{P}>0.05)^{26}$.

At six weeks post intervention, contraceptive knowledge was statistically significant between the two groups. The intervention group had higher contraceptive knowledge scores than the comparison group in all the 6 domains of the knowledge domain. Respondents in the intervention group were five times more knowledgeable than those in the comparison group $(\mathrm{p}<0.05)$. This is because the intervention group was exposed to postpartum family planning counselling and showed that as contraceptive counselling increases, so does contraceptive knowledge and the likelihood that respondents would scale up contraceptive uptake better. This further indicates that the intervention offered to women in the intervention group was effective in improving post-partum women's contraceptive knowledge. This supports the need to provide and sustain this counseling offered to women post partum The above, findings are consistent with a Zimbabwean study on contraceptive counselling among HIV positive mothers, which showed that a significant difference in knowledge existed between the intervention and comparison groups at 3 months with $85.5 \%$ of the intervention, and $56.3 \%(\mathrm{P}<0.002)$ in the comparison $\operatorname{group}^{26}$.

\section{Attitude towards Contraceptives Usage}

In this study, at baseline, contraceptive attitude was not statistically significant between the two groups. This showed that the two groups had similar contraceptive attitudes at baseline. This is in line with a study among postpartum women which revealed that at baseline there was no significant difference between the intervention and comparison group in terms of contraceptives 
attitude in Iran $(\mathrm{p}>0.05)^{15}$. Interestingly, at posttest, contraceptive attitude was statistically significant between the two groups. The intervention group had more positive contraceptive attitude scores than the comparison group in all the 9 domains of the attitude subscales. Respondents in the intervention group had about three times more positive attitude towards contraceptives than those in the comparison group. This is because the intervention group was exposed to postpartum family planning counseling. This showed that the post-partum contraceptive counseling offered to women in the intervention group improved women's attitude towards contraceptives and indicated that the intervention was effective in improving postpartum women's attitude towards contraceptives. By this result, it is concluded that, there was significant effect of the intervention (Postpartum Family Planning Counselling) on women's attitude towards contraceptive.

This finding falls in line with a study among postpartum women in Iran which revealed that the intervention group differed significantly from the comparison group in terms of contraceptives attitude $(\mathrm{p}<0.05)$ posttest $^{15}$. Similarly, a study also revealed that most postpartum women had favorable attitude toward contraceptives in Ethiopia ${ }^{25}$.

\section{Intention towards Contraceptives Usage}

At baseline, intention towards contraceptives did not differ significantly between the two groups. Both groups had the same low intention towards contraceptives. In Uganda researchers found that there was no significant difference in women's intention to use contraceptive at baseline ${ }^{16}$.

At six weeks posttest, a statistically significant difference existed in women's intention between the intervention and comparison groups $(\mathrm{p}<0.05)$. The intervention group had higher intention 
contraceptive scores than the comparison group in all the 9 domains of the intention subscales. Similarly, the mean contraceptive intention score was higher in the intervention group than the comparison group. Furthermore, respondents in the intervention group had about 36 times higher intention to contraceptives than those in the comparison group. There was statistically significant between the two groups. This difference can be accounted for because the intervention group was exposed to postpartum family planning counselling and it shows that the post-partum contraceptive counselling offered to women in the intervention group was able to improve women's intention towards contraceptive.

A study conducted in Uganda revealed that $71.4 \%$ of women in the comparison and $87 \%$ in the intervention had intention to use a modern contraceptive method following counselling on postpartum family planning and the difference was statistically significant ${ }^{16}$. One study from Pakistan, found that at 8-12 weeks postpartum all women in the counselling group planned to use a modern contraceptive method compared with only a third in the comparison group ${ }^{27}$. Within the intervention study group, all the 8 items of the intention subscale were found to be statistically significant $(\mathrm{p}<0.05)$. This shows that significant gain in intention was registered from pretest to posttest.

\section{Conclusion}

Effective family planning counseling is one of the cornerstones for increasing contraceptive acceptance and use, during the postpartum period. Findings of this study indicated that at post intervention participants had better knowledge, more positive attitudes, and higher intentions towards contraceptive uptake. These were found to be statistically significant. The present study therefore highlights the need to conduct family planning counseling during the immediate 
postpartum period, particularly before discharge of women from the hospital so as to ensure improve contraceptive uptake. This would curb unwanted pregnancies, unsafe abortions, improve birth spacing and reduce the high fertility rate and maternal mortality in the country. All these would improve maternal health outcomes and help to achieve the sustainable development goals 3 and 5 .

\section{LIST OF ABBREVIATIONS}

CPR: $\quad$ Contraceptive Prevalence Rate

CI Confidence Interval

BGH: $\quad$ Bansang General Hospital

FP: $\quad$ Family Planning

GATHER Great, Ask, Tell, Help, Explain, Return

HIV Human Immunodeficiency Virus

PPFP: $\quad$ Postpartum Family Planning

SHC Soma Health Center 


\section{References}

1. WHO. Programming Strategies for Postpartum Family Planning. Geneva: Available 2013. http://www.who.int/reproductivehealth/publications/family_planning/ppfp_strategies/en/ Accessed October $4^{\text {th }}, 2016$

2. USAID. Postpartum Family Planning Toolkit' https://www.k4health.org/toolkits/ppfp 2016. Accessed $11^{\text {th }}$ November, 2018.

3. Singh, S., \& Darroch, J. E. Adding it up: costs and benefits of contraceptive services estimates for 2012. New York: Guttmacher Institute and United Nations Population Fund (UNFPA) 2012. http://www.guttmacher.org/pubs/AIU-2012-estimates.pdf .Accessed 01 ${ }^{\text {st June, } 2016 .}$

4. Ministry of Health and Family Welfare, Government of India. 2010. Postpartum IUCD Reference Manual, Family Planning Division, New Delhi, India

5. Borda, M.R., \& Winfrey, W. Postpartum fertility and contraception: an analysis of findings from 17 countries. Baltimore, MD: Jhpiego. 2010. Available from: http://www.k4health.org/toolkits/lam/postpartum-fertility-and-contraception-analysisfindings-17-countries. Accessed $26^{\text {th }}$ January, 2016

6. The Gambia Demographic and Health Survey. Gambia Bureau of Statistics, Banjul, The Gambia, 2013.

7. Kaunitz, A.M. Contraceptive counselling and selection . 2018. https://www.uptodate.com/contents/contraceptive-counseling-and-selection. Accessed $28^{\text {th }} /$ October, 2018.

8. El-Khoury, M., Thornton, R., Chatterji, M., Kamhawi, S., Sloane, P., \& Halassa, M. Counseling women and couples on family planning: A randomized study in Jordan. Studies in Family Planning, 2016; 47 (3), 222-238.

9. Lemani, C., Tang, J. H., Kopp, D., Phiri, B., Kumvula, C., Chikosi, L., Mwale, M., \& Rosenberg, N.E.. Contraceptive uptake after training community health workers in couples counseling: A cluster randomized trial. PLoS one, 2017; 12 (4), e0175879 1-10.

10. Davalagi, S., Revanna, R., \& Agadi, N. Determinants of Postpartum contraception practices in urban slums of central Karnataka, India. Indian Journal of Community Health, 2016; 28 (3), 280-285

11. American College of Obstetricians and Gynecologists. Practice Bulletin No. 121: 
Long-acting reversible contraception: implants and intrauterine devices. Obstetrics \& Gynecology, 2011; 118 (1), 184-196

12. Monjok, E., Smesny, A., Ekabua, K. E., \& Essien J. Contraceptive practices in Nigeria: Literature review and recommendation for future policy decisions. Open Access Journal of Contraception, 2010; 1, 9-22.

13. Bajracharya, A. Knowledge, attitude and practice of contraception among postpartum women attending Kathmandu Medical College Teaching Hospital.

Kathmandu University Medical Journal, 2015; 13 (52), 292-297

14. Anaba, R., Ugwa, E. A., Agbor, I. E., Nwali, M. I., \& Orji, B. Knowledge, attitude, and contraceptive preferences among postpartum women in Izzi, Ezza South, and Ikwo Local Government Areas of Ebonyi State, Nigeria. Hospital Practice Research, 2018; 3 (1), 1115.

15. Mahamed, F., Parhizkar, S. \& Raygan Shirazi, A.. Impact of family planning health education on the knowledge and attitude among Yasoujian women. Global Journal of Health Science, 20124 (2), 110-118.

16. Ayiasi, R. M., Muhumuza, C., Bukenya, J., \& Orach, C. G. The effect of prenatal counselling on postpartum family planning use among early postpartum women in Masindi and Kiryandongo districts, Uganda. PanAfrican Medical Journal, 2015; 21, 138.

17. Ujah O. I., Ocheke A. N., Mutihir, J. T., Ojopi, J. A., \& Ujah, I. A. O.. Postpartum Contraception: determinants of intention and methods of use among an obstetric cohort in a tertiary hospital in Jos, North Central Nigeria. International Journal of Reproductive Contraception, Obstetrics \& Gynecology, 2017; 6 (12), 5213-5218.

18. Weisband, Y.L., Keder, L.M., Sarah A. Keim, S.A., \& Gallo, M. F. Post-partum Intentions on contraception use and method choice among breastfeeding women attending a university hospital in Ohio: a cross-sectional study. Reproductive Health, 2017; 14 (1), 45-52.

19. Charan, J., \& Biswas, T. How to calculate sample size for different study designs in medical research? Indian Journal of Psychological Medicine, 2013; 2: 121-126

20. Dulli, L.S., Eichleay, M., Rademacher, K., Sortijas, S., \& Nsengiyumva, T. Meeting postpartum women's family planning needs through integrated family planning and immunization services: Results of a cluster-randomized controlled trial in Rwanda. Global Health, Science and Practice, 2016; 4 (1), 73-86. 
21. Rinehart, W., Rudy, S., Drennan, M. GATHER guide to counselling. Population Report Journal, 1998; 48:1-31.

22. Shabiby, M.M., Karanja, K.G., Odawa, F., Kosgei, R., Kibore, M.W., Kiarie, J.N., \& Kinuthia, J. Factors influencing uptake of contraceptive implants in the immediate postpartum period among HIV infected and uninfected women at two Kenyan District Hospitals. BioMed Central Women's Health, 2015; 15 (62), 1-8.

23. Choudhary, D., Pal, R., \& Goel, N. Awareness and practice patterns of family planning methods. Biomed Journal, 2015; 38 (4), 356-358.

24. Colley, S, Kao, C., Gau, M., \& Cheng, S.. Women's perception of support and control during childbirth in The Gambia, a quantitative study on dignified facility-based intrapartum care. BioMed Central Pregnancy and Childbirth, 2018; 18 (413), 1-9.

25. Abraha, T.H., Balay, H. S., \& Welay, G.M. Intentions on contraception use and its associated factors among postpartum women in Aksum town, Tigray region, northern Ethiopia: a community-based cross- sectional study. Reproductive Health, 2018; 15 (188), 1-8.

26. Sarnquist, C.C., Moyo, P., Stranix-Chibanda, L., Chipato, T., Kang, J.L., \& Maldonado, Y.A. (2014). Integrating family planning and prevention of mother to child HIV transmission in Zimbabwe. Contraception, 2014; 89 (3), 209-214.

27. Dhadwal, V. Education for contraceptive use by women after childbirth: RHL. The WHO Reproductive Health Library; Geneva: World Health Organization. 2012

\section{Acknowledgement}

Special thanks are due to the Gambia Government (GG) through the Ministry of Higher

Education, Research, Science and Technology and World Bank under the African Center of

Excellence (ACE) Scholarship Project for awarding the first author with the scholarship to pursue a Doctor of Philosophy Degree in Reproductive and Family Health Nursing.

\section{Funding}

The study was part of a Ph.D. Thesis funded by the Gambia Government in partnership with the World Bank, African Center of Exellence Scholarship Project.

\section{Contributions}


JSS conceptualized the topic, wrote the proposal and draft report. FO Adeyemo and H.O Okogie participated in data analysis and proof read the final report. All authors read, and approved the manuscript for publication. '

\section{Ethical Approval and Consent to Participate}

Ethical approval was granted by the Research and Ethic Committee, School of Basic Medical Sciences, College of Medicine, University of Benin (CMS/REC/2017/017); The Gambia Government/Medical Research Council Laboratories Joint Ethics Committee (R017016Av1.1). Participants gave informed consent and later appended thumbprints to the consent form indicating approval. This was allowed by the above ethic committees, since majority could neither read nor write in English Language and could not signed the consent from. Participants were assured that participation was voluntary, and they had the right to withdraw from the study at any time. This would not affect their care or services offered. Data was pooled and used only for the study.

\section{Competing Interests}

The authors declare that they have no competing interests.

\section{Availability of Data and Materials}

The datasets used to analyze this study are available from the corresponding author on genuine request.

\section{Consent for Publication}

All authors consented for this paper to be published 
Figures

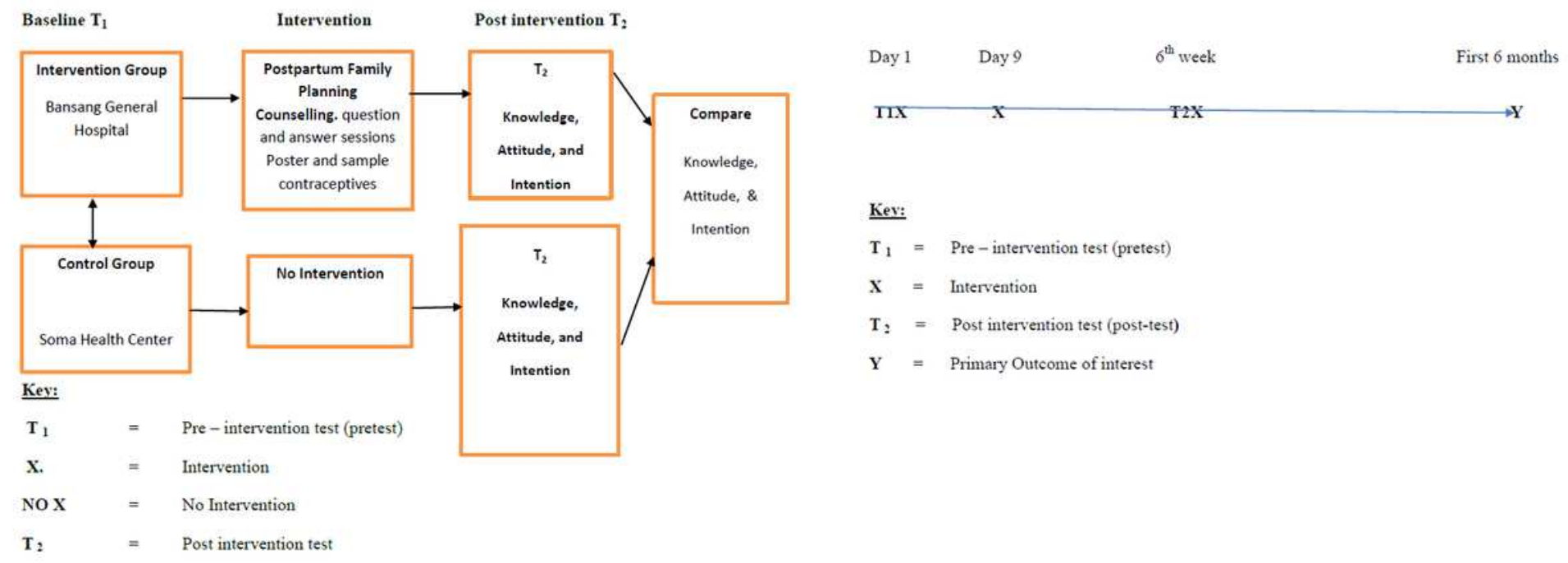

\section{Figure 1}

1.1: Study Design 1.2: Intervention Plan.

\section{Supplementary Files}

This is a list of supplementary files associated with this preprint. Click to download.

- ManuscriptTablesREV.pdf 